\title{
NON-UNIFORM CELLULAR AUTOMATA
}

\section{Marifi GÜLER ${ }^{1}$ and Hürevren KILIÇ ${ }^{2}$}

1Department of Computer Engineering, Middle East Technical University,06531 Ankara, Türkiye

2Bilkent Computer Center, Bilkent University, Bilkent,06533 Ankara, Türkiye

Abstract- A model called Non-uniform Cellular Automata (NCA) is introduced and its capability to do mapping and computation is studied. The suggested model is similar to the original cellular Automata, with its local neighborhood property, but neighborhood definition of cells do change in space and time (non-uniform) depending on the input-output template pairs introduced during the training phase.

Neighborhood degree of a cell can be defined as the number of neighbors that this cell uses to specify its next state. Only the cells having the same neighborhood degree have to evolve according to the same transition function rules. In other words, different neighborhood degrees may cause different transition functions.

NCA has two modes of operation similar to neural networks: training and mapping. At the beginning of the training mode, neighborhood of a cell is only itself. Neighborhood degree and transition functions for each cell gradually develop in accordance with the input patterns and the corresponding output bit desired to be mapped. At the end of the training, ultimate neighborhoods and cell functions are defined and one can introduce any input template to the system and get its intended output template. NCA can simply be considered as a parallel mapping system which makes its decision in one step of evolution.

In this paper, we have considered one-dimensional circularly connected cells. The length of an input template is the same as the length of an output template and is equal to the number of cells in the system. The algorithm used allows a cell to increase its neighborhood until all the templates are properly mapped. If the graph of the trained system has a strong component then the system will do computation.

The look-up table search and the lack of generalization are drawbacks to NCA. However, among some other possible ways of overcoming those drawbacks, relating the model to neural networks is discussed. Considering cells as neurons is one suggestion. 